\title{
Friedrichs, Werner, und Sebastian Hamm (Hrsg.) (2020): Zurück zu den Dingen! Politische Bildungen im Medium gesellschaftlicher Materialität
}

\author{
Baden-Baden: Nomos. 423 Seiten. $59 €$
}

\section{Claire Moulin-Doos}

Angenommen: 27. September 2021 / Online publiziert: 14. Oktober 2021

(C) Der/die Autor(en) 2021

„In den politischen Bildungen materialisiert und artikuliert sich das Politische“ (S. 15) ist in der Einleitung des vorliegenden Sammelbandes zu lesen. Politische Bildung ist nicht irgendein (Schul-)Fach, sondern sollte für alle Sozialwissenschaftler und nicht nur für die Politikdidaktiker unter ihnen von großem Interesse sein. Die zentrale Problematisierung bzw. das Anliegen des Bandes besteht darin, politische Bildungen jenseits des Subjekt/Objekt-Dualismus der Moderne bzw. des idealistischen Liberalismus zu schaffen, also einen Dualismus, in dem ein Mensch als erkennendes „Subjekt“ gegenüber der Welt als passives „Objekt“ stehen würde, um über diese distanzierte Welt nachzudenken. Der Beitrag des Mitherausgebers Werner Friedrichs könnte gerade nach der Einführung gelesen werden, da er als vertiefende Einführung zum intendierten Projekt des Bandes wirkt: Bildungen werden neumarxistisch gedacht, also im Sinne eines weiterentwickelten Marxismus, der von seinem Idealismus freigemacht wird, um der Materialität, den Dingen, gerecht zu werden (S. 180).

„Dinge“ werden in der politischen Bildung bereits thematisiert. Jedoch werden sie immer noch nur als Objekte behandelt, als passive Wesen verstanden: Dinge, Sachen, Objekte werden von Menschen benutzt und behandelt, im schlimmsten Fall ausgebeutet und zerstört oder im besten Fall gepflegt. Dank dem ,,material turn“, der Akteur-Netzwerk-Theorie (ANT) und dem neuen (ent-idealisierten) Materialismus - allesamt Ansätze, die in diesem Band erläutert werden - kehren diese verdrängten Objekte zurück. Mit u. a. Bruno Latour werden die Dinge als aktive Wesen bzw. als Aktanten erkannt. In der Politikdidaktik ist dieser ,,material turn“ jedoch noch nicht angekommen. Mündigwerden soll nicht mehr außerhalb der Welt gedacht werden, sondern zusammen mit Dingen in unserer Beschaffenheit als terrestrische Wesen.

Claire Moulin-Doos $(\varangle)$

Universität Kassel, Kassel, Deutschland

E-Mail: moulindoos@uni-kassel.de 
Wir werden durch und mit Dingen politisch bzw. politisch gebildet. In diesem Zusammenhang denkt Martin Repohl mithilfe von Hartmut Rosa die Subjektivierung durch „Dingresonanz“ (S. 101), indem Subjekte sich in Beziehung zu anderen Subjekten und zur Welt konstituieren: Diese Gedanken können sehr produktiv werden, insbesondere in Bildungskontexten, die immer noch das Subjekt von seinen Abhängigkeiten befreien möchten, anstatt Freiheit innerhalb dieser Beziehungen zu konzipieren. Carsten Bünger und Kerstin Jergus denken auch Subjektivierungsprozesse in und entlang der Bezogenheit auf Dingen (S. 154). Dinge sind nicht per se politisch oder unpolitisch, dennoch können sie in Politisierungsprozessen wechselseitig mit Subjekten eingebunden werden. Die Autoren greifen das Kopftuch als Beispiel auf. Das Kopftuch ist nicht inhärent politisch, es kann und wird in einigen Kontexten politisiert (S. 155). In der (politischen) Bildung sollte die performative Dimension von „Ding-Praktiken“ (S. 156) berücksichtigt werden zusammen mit der „Wechselwirkung“ (S. 161) von Dingen, die nicht eindeutig vor-determiniert vor uns liegen, und Subjekten, die nicht in einer abgeschlossenen Identität existieren, sondern nur in Verhältnissen zu anderen (Menschen und Dingen). Das Subjekt des Bildungsprozesses sei ,keine Entität, die vor und nach dem Bildungsprozesse in holistischer Weise sich selbst transparent gemacht werden könnte“ (S. 162). Passend zu diesem Beitrag sind auch die Beiträge von Gustav Roßler über Dingpolitik sowie Simon Clemens und Marco Schmandt zur Aktualisierung politischer Öffentlichkeit. Im letztgenannten wird von „Quasi-Subjekten“ und „Quasi-Objekten“ (S. 135) als Akteuren politischer Öffentlichkeit gesprochen, die auch diese Annäherung zwischen Menschen und Dingen im Sinne von Latour ausdrücken. Nicht das autonome Subjekt handelt, sondern Handeln ist ein „Knoten“ (S. 136), es sind Netzwerke von Aktanten, die gemeinsam als Netzwerk handeln. In der politischen Bildung sollten daher Quasi-Objekte bzw. nichtmenschliche politische Aktanten als solche sichtbar gemacht werden und für diese sensibilisiert werden (S. 143). Die Quasi-Subjekte Schüler sollten befähigt und ermächtigt werden, diese Phänomene besser verstehen zu können (S. 144). Bildungsarbeit wird an dieser Stelle sehr kognitiv verstanden, wobei Klang (Kerstin Meißner), Sound (Alfred Schäfer) oder Kunst (Iris Clemens und Christian Heilig) andere Dimensionen als die kognitive mobilisieren könnten, um den Dingen politisch gerecht zu werden und besonders bei der jüngeren Generation didaktisch produktiv wirken zu können.

Was die Technik betrifft, so bietet der Band zahlreiche Analysen und Beispiele für ihre Mit-Wirksamkeit. Dinge mit Alltagsnutzen wie ein Fahrrad (Olaf Sanders) oder ein Auto (Friedrichs) sind didaktisch sehr gut dafür geeignet. Die Guillotine (David Salomon) hat den König enthauptet und damit die Transzendenz der Herrschaft im (zumindest französischen) politischen Denken abgeschafft, ob sie die deutschen Politikdidaktiker zum ,,material turn“ bewegen wird, ist eher fraglich. Insbesondere legt dieser Band viele Einblicke in Medien vor, einem Thema, das bereits umfangreich in der politischen Bildung behandelt wird. Hier können die jeweiligen Beiträge über Medien als öffentliche Dinge (Roßler), Nudges (Sönke Ahrens), Fake News (Johanna Taufner) sehr gut an bereits vorhandene fachdidaktische Ansätze über Medien anknüpfen. Der anspruchsvolle Beitrag von Roger Häußling, welcher zugleich Wirtschaftsingenieur und Soziologe ist, ist insbesondere für Sozialwissenschaftler lesenswert, um die technische Logik der digitalen „Datafizierung“ (S. 58), 
wie es sich die Herausgeber zu Recht wünschen, nicht allein den Informatiker zu überlassen (S. 18). Mithilfe von Karen Barad (relationaler Materialismus) und Harrison White (Netzwerktheorie) stellt Häußling vor, wie in der digitalen Gesellschaft keine „Repräsentation“, sondern „Relationierung“ (S. 54) stattfindet. Genauer gesagt hilft uns der Beitrag, einen Blick in die Logik der Aussortierung durch Algorithmen und der Sinnerzeugung mittels Daten zu werfen. Durch die Kombinierbarkeit der Daten, dank ,wechselseitige[r] Beeinflussung algorithmischer und sozialer Prozesse“ (S. 58) können „neue Sinngehalte“ (S. 59) produziert werden. Daten sind dadurch als „Produkte von Relationierungen“ (S. 58) zu verstehen. In Lehr-LernSituationen hört man regelmäßig: „Ich hab's gegoogelt“, als ob die Ergebnisse nicht auf komplexe Algorithmen gestützt sind, sondern eine reine „Faktensuche“ nach „Daten“ seien. Die Relationierung von der durch Algorithmen erzeugten Auswahl von Daten und der soziale Prozess u. a. innerhalb der Schulklasse schafft neue soziale Wirklichkeiten. Es wird zu wenig wahrgenommen, dass Medien mitgestalten, also Aktanten sind, wo doch die politische Wirksamkeit der Medien in der politischen Bildung bereits embryonal vorhanden ist. Insgesamt werden Medien didaktisch immer noch hauptsächlich als Mittel zum Zweck verstanden, was sich nach Darstellung des Autors darin zeigt, dass unter Digitalisierung von Schulen oft ausschließlich die Ausstattung der Schulen mit PCs und Whiteboards gemeint wird (S. 57).

Die politische Wirksamkeit von Dingen sollte in der politischen Bildung forciert und generalisiert werden, sowohl wie gerade erläutert für Medien als auch für die Wirksamkeit von anderen Dingen. Im vorliegenden Band werden vor allem technische Dinge bedacht, unterrepräsentiert hingegen verbleibt die Natur, verstanden u. a. als Wälder, Tiere, Ozeane, Viren usw. Dennoch werden wertvolle Ansatzpunkte geboten, um die politische Bildung hin zum Anthropozän zu führen, um endlich das veraltete Konzept der Bildung für Nachhaltige Entwicklung (BNE) hinter sich lassen zu können.

Dieses Buch ist ein willkommener Baustein für einen Weg der Ent-Idealisierung bzw. Wieder-Materialisierung von politischen Bildungen, in dem Versuch, endlich in den politisch-demokratisch-erzieherischen „Immanentismus“ (S. 174) einzutreten und nicht mehr in einem erhofften kantischen Blick über die Welt zu verharren, sondern sich ,im performativen Weltkontakt“ (S. 14) zu bilden. Die politische Bildung wird dennoch viel mehr brauchen als dieses Buch, um den Dingen gerechter zu werden, vor allem auch für Politikdidaktiker zugänglicher geschriebene Werke, denn das richtige Maß an didaktischer Reduktion gibt es in abstracto nicht.

Dinge sind besonders bei den Grundschülern sehr präsent, nicht zuletzt innerhalb des ,Sach“unterrichts. Nur die Art und Weise, wie diese bis jetzt bearbeitet werden, ist dem „material turn“ nicht angemessen. Mit diesem könnte der Sachunterricht „Sache“ nicht nur aus der Perspektive vom Ich-Nutzer oder vom Ich-Beschützer der Sache thematisieren. Vielmehr könnte Sachunterricht dazu bilden, mit Dingen gemein zu werden, also zusammen zu leben. Statt das Ich in den Mittelpunkt zu stellen, könnte der „Sach“unterricht (und nicht „Ich“unterricht) und insbesondere die sozialwissenschaftliche Perspektive innerhalb des Sachunterrichts eine Dezentrierung des Ichs und eine Öffnung zu einem Wir, Menschen und Dinge oder ,WirNetzwerke“ (Roßler, S. 36) oder zu ,der öffentlichen Sache“ (Roßler, S. 36) vorantreiben. Unsere Abhängigkeiten sollten wahrgenommen und reflektiert und nicht 
als (idealistisch verstandene) „Unfreiheit“ negiert werden. Diese Abhängigkeiten sind überhaupt erst als Voraussetzung (politischer) Freiheit zu erfassen. Anstatt der „,moderne[n] bürgerliche[n] Vorstellung von Bildung“ mit ihrem Anspruch zur ,Entfaltung der Individualität“ (Bünger und Jergus, S. 160) zu hofieren, sollte man gegen den Strich viel mehr nach dem Motto: „Das Politische ebenso wie Bildung haben es mit Materialisierungen eines Immateriellen zu tun, sie gehen einher mit Identifizierungen, ohne jedoch in Identitäten zu münden“"(Bünger und Jergus, S. 162) handeln.

Funding Open Access funding enabled and organized by Projekt DEAL.

Open Access Dieser Artikel wird unter der Creative Commons Namensnennung 4.0 International Lizenz veröffentlicht, welche die Nutzung, Vervielfältigung, Bearbeitung, Verbreitung und Wiedergabe in jeglichem Medium und Format erlaubt, sofern Sie den/die ursprünglichen Autor(en) und die Quelle ordnungsgemäß nennen, einen Link zur Creative Commons Lizenz beifügen und angeben, ob Änderungen vorgenommen wurden.

Die in diesem Artikel enthaltenen Bilder und sonstiges Drittmaterial unterliegen ebenfalls der genannten Creative Commons Lizenz, sofern sich aus der Abbildungslegende nichts anderes ergibt. Sofern das betreffende Material nicht unter der genannten Creative Commons Lizenz steht und die betreffende Handlung nicht nach gesetzlichen Vorschriften erlaubt ist, ist für die oben aufgeführten Weiterverwendungen des Materials die Einwilligung des jeweiligen Rechteinhabers einzuholen.

Weitere Details zur Lizenz entnehmen Sie bitte der Lizenzinformation auf http://creativecommons.org/ licenses/by/4.0/deed.de. 